\title{
The Limit Deposit Velocity model, a new approach
}

\author{
Sape A. Miedema ${ }^{1 *}$, Robert C. Ramsdell ${ }^{2}$ \\ ${ }^{1}$ Delft University of Technology, Mekelweg 2, 2628 CD Delft, The Netherlands. \\ ${ }^{2}$ Manager Production Department, Great Lakes Dredge \& Dock, 2122 York Road, Oak Brook, IL 60523 USA. \\ ${ }^{*}$ Corresponding author. Tel.: +31-15-2788359. E-mail: s.a.miedema@tudelft.nl
}

\begin{abstract}
In slurry transport of settling slurries in Newtonian fluids, it is often stated that one should apply a line speed above a critical velocity, because blow this critical velocity there is the danger of plugging the line. There are many definitions and names for this critical velocity. It is referred to as the velocity where a bed starts sliding or the velocity above which there is no stationary bed or sliding bed. Others use the velocity where the hydraulic gradient is at a minimum, because of the minimum energy consumption. Most models from literature are one term one equation models, based on the idea that the critical velocity can be explained that way.

Here the following definition is used: The critical velocity is the line speed below which there may be either a stationary bed or a sliding bed, depending on the particle diameter and the pipe diameter, but above which no bed (stationary or sliding) exists, the Limit Deposit Velocity (LDV). The way of determining the LDV depends on the particle size, where 5 regions are distinguished.

These regions for sand and gravel are roughly; very small particles up to $0.014-0.040 \mathrm{~mm}\left(d<\delta_{v}\right)$, small particles from $\delta_{v}-0.2 \mathrm{~mm}$, medium particles in a transition region from $0.2-2.00 \mathrm{~mm}$, large particles $>2 \mathrm{~mm}$ and very large particles $>0.015 \cdot D_{p}$. The lower limit of the LDV is the transition between a sliding bed and heterogeneous transport. The new model is partly based on physics and correlates well with experiments from literature.
\end{abstract}

Keywords: Critical velocity; Limit Deposit Velocity; Slurry transport; Newtonian fluid.

\section{INTRODUCTION}

The Delft Head Loss \& Limit Deposit Velocity Framework (DHLLDV) is a framework based on constant spatial volumetric concentration curves and uniform sands or gravels for 5 flow regimes in a Newtonian fluid (Miedema and Ramsdell, 2014a); (Ramsdell and Miedema, 2013). These 5 regimes are the stationary or fixed bed regime (Miedema and Matousek, 2014) the sliding bed regime (Miedema and Ramsdell, 2014b), the heterogeneous regime (Miedema, 2014), (Miedema, 2015c) and (Miedema and Ramsdell, 2013), the homogeneous regime (Miedema, 2015a) and the sliding flow regime (Miedema and Ramsdell, 2014a). Crucial for the modelling is the determination of the Limit Deposit Velocity (LDV), which is the topic of this paper. The constant delivered volumetric concentration curves are determined based on the LDV and the holdup function (Miedema, 2015b). The bed height is determined based on the LDV and the holdup function. Curves for graded sands and gravels are determined by superposition of the curves of the fractions of the Particle Size Distribution (PSD).

The Limit Deposit Velocity is defined here as the line speed where there is no stationary bed or sliding bed, Thomas (1962). Below the LDV there may be either a stationary or fixed bed or a sliding bed.

For the critical velocity often the Minimum Hydraulic Gradient Velocity (MHGV) is used, Wilson (1942). For higher concentrations this MHGV may be close to the LDV, but for lower concentrations this is certainly not the case. Yagi et al. (1972) reported using the MHGV, making the data points for the lower concentrations to low, which is clear from Fig. 1.

Another weak point of the MHGV is, that it depends strongly on the model used for the heterogeneous flow regime. Durand and Condolios (1952), Fuhrboter (1961), Jufin and Lopatin (1966) and others will each give a different MHGV. In dredging the process is instationary, meaning a constantly changing PSD and concentration in long pipelines, making it almost impossible to determine the MHGV.

Wilson (1979) derived a method for determining the transition velocity between the stationary bed and the sliding bed, which is named here the Limit of Stationary Deposit Velocity (LSDV). Since the transition stationary bed versus sliding bed, the LSDV, will always give a smaller velocity value than the moment of full suspension or saltation, the LDV, one should use the LDV, to be sure there is no deposit at all. For small particles it is also possible that the bed is already completely suspended before the bed could ever start sliding (theoretically). In that case an LSDV does not even exist.

This is the reason for choosing the LDV as the critical velocity and developing a new model for this, independent of the head loss model and always existing.

The Froude number $F_{L}$ is often used for the LDV, because it allows comparison of the LDV for different pipe diameters $D_{p}$ and relative submerged densities $R_{s d}$ without having to change the scale of the graph, this is defined as:

$$
F_{L}=\frac{v_{l s, l d v}}{\sqrt{2 \cdot g \cdot R_{s d} \cdot D_{p}}}
$$

It should be noted that sometimes the 2 and sometimes the 2 and the relative submerged density $R_{s d}$ are omitted.

Because there are numerous data and equations for the critical velocity (LSDV, LDV or MHGV), some equations based on physics, but most based on curve fitting, a selection is made of the equations and methods from literature. The literature analysed are from Wilson (1942), Durand and Condolios (1952), Newitt et al. (1955), Jufin and Lopatin (1966), Zandi and Govatos (1967), Charles (1970), Graf et al. (1970), Wilson and Judge (1976), Wasp et al. (1977), Wilson and Judge (1977), Thomas (1979), Oroskar and Turian (1980), Parzonka et al. 
(1981), Turian et al. (1987), Davies (1987), Schiller and Herbich (1991), Gogus and Kokpinar (1993), Gillies (1993), Berg (1998), Kokpinar and Gogus (2001), Shook et al. (2002), Wasp and Slatter (2004), Sanders et al. (2004), Lahiri (2009), Poloski et al. (2010) and Souza Pinto et al. (2014).

The research consisted of two parts, analyzing the experimental data and analyzing the resulting models based on these data. First the experimental data will be discussed.

\section{EXPERIMENTAL DATA}

Fig. 1 shows many data points of various authors for sand and gravel in water. Each column of data points shows the results of experiments with different volumetric concentrations, where the highest points were at volumetric concentrations of about $15 \%-20 \%$, higher concentrations gave lower points. The experimental data also shows that smaller pipe diameters, in general, give higher Durand and Condolios (1952) Froude $F_{L}$ numbers. The two curves in the graph are for the Jufin and Lopatin (1966) equation, which is only valid for sand and gravel, and the DHLLDV framework which is described in this paper.

Both models give a sort of upper limit to the LDV. The data points of the very small particle diameters, Thomas (1979) and Poloski (2010), were carried out in very small to medium diameter pipes, while the two curves are constructed for a 0.1524 (6 inch) pipe, resulting in slightly lower curves. Data points above the DHLLDV curve are in general for pipe diameters smaller than $0.1524 \mathrm{~m}$. Some special attention is given to the relation between the Durand Froude $F_{L}$ number and the pipe diameter $D_{p}$. Both Thomas (1979) and Wasp et al. (1977) carried out research with a $d=0.18 \mathrm{~mm}$ particle in 6 pipe diameters, see Fig. 2. These experiments show a slight decrease of the $F_{L}$ value with increasing pipe diameter with a power close to -0.1 .

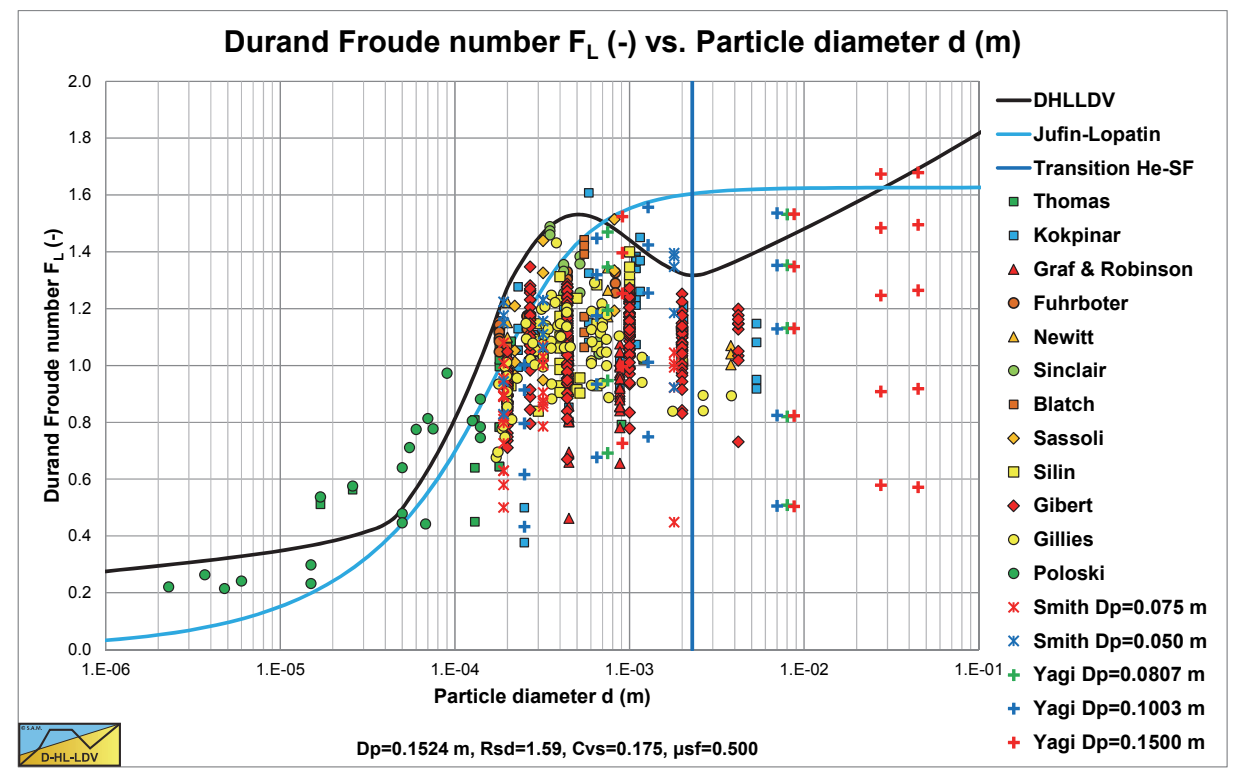

Fig. 1. LDV and MHGV (Yagi) data of many authors.

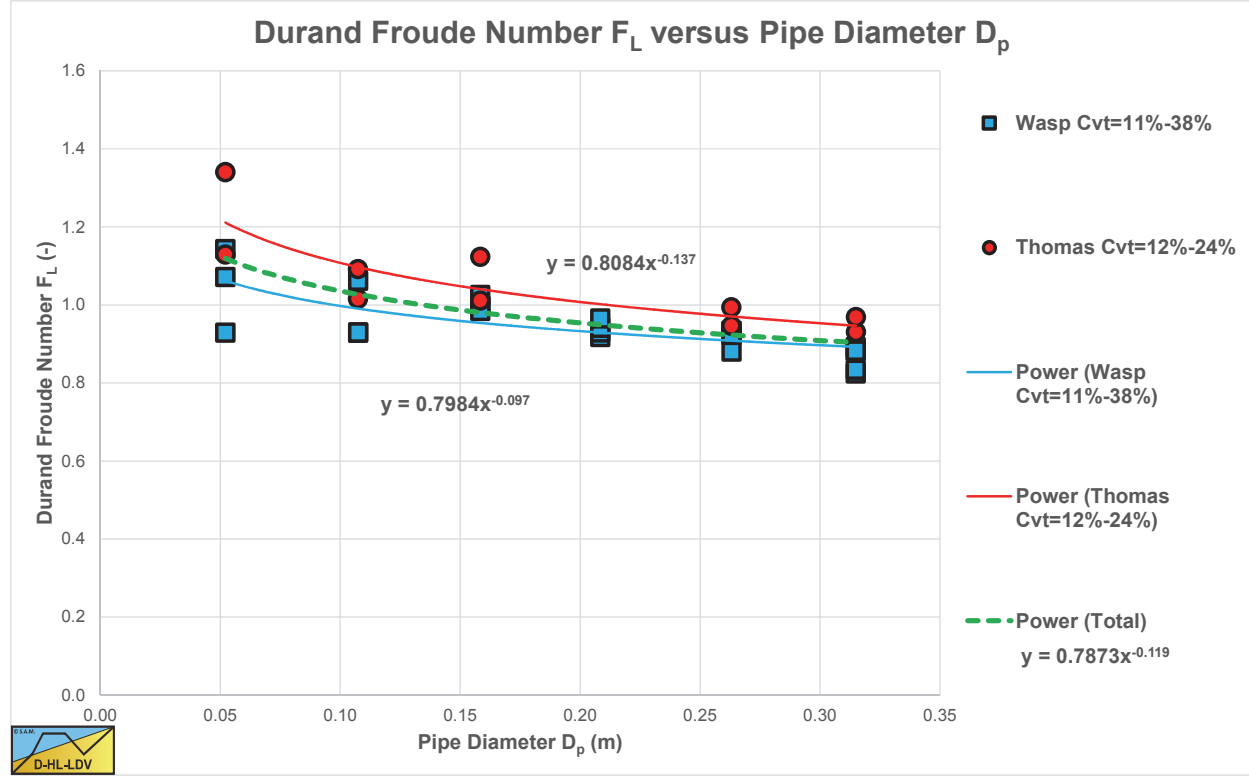

Fig. 2. Durand Froude $F_{L}$ number versus pipe diameter $D_{p}$. 


\section{EQUATIONS \& MODELS}

Fig. 3 and Fig. 4 show the Limit Deposit Velocities of DHLLDV, Durand and Condolios (1952), Jufin and Lopatin (1966), Wasp et al. (1970), Wasp and Slatter (2004), Souza Pinto et al. (2014), Hepy et al. (2008), Gogus and Kokpinar (1993), Kokpinar and Gogus (2001), Berg (1998), Turian et al. (1987) and Gillies (1993) for 2 pipe diameters. The curves of Hepy et al. (2008), Gogus and Kokpinar (1993) and Kokpinar and Gogus (2001) show a maximum $F_{L}$ value for particles with a diameter near $d=0.5 \mathrm{~mm}$. However these models show an increasing $F_{L}$ value with the pipe diameter, which contradicts the numerous experimental data, showing a slight decrease. The models of Turian et al. (1987), Wasp et al. (1970), Wasp and Slatter (2004) and Souza Pinto et al. (2014) show an increasing $F_{L}$ value with increasing particle diameter and a slight decrease with the pipe diameter.
Jufin and Lopatin (1966) show an increase with the particle diameter and a slight decrease with the pipe diameter (power 1/6). The model of van den Berg (1998) shows an increasing $F_{L}$ with the particle diameter, but no dependency on the pipe diameter. Durand and Condolios (1952) did not give an equation but a graph. The data points as derived from the original publication in (1952) (Low Correct) and from Durand (1953) (High Incorrect) are shown in the graphs. The data points show a maximum for $d=0.5 \mathrm{~mm}$. They did not report any dependency on the pipe diameter. The model of Gillies (1993) tries to quantify the Durand and Condolios (1952) data points (the incorrect ones) but does not show any dependency on the pipe diameter for the $F_{L}$ Froude number. The increase of the $F_{L}$ value with the pipe diameter of the Hepy et al. (2008), Gogus and Kokpinar (1993) and Kokpinar and Gogus (2001) models is probably caused by the forced $d / D_{p}$ relation.

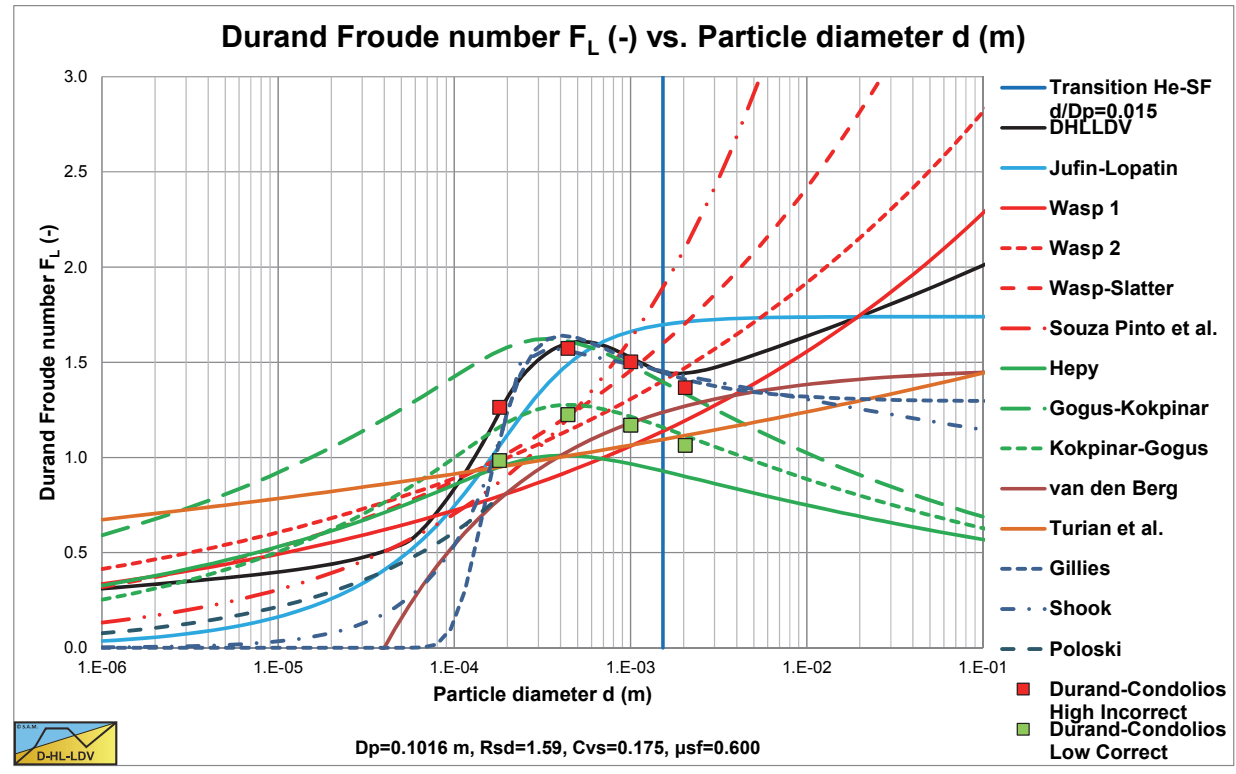

Fig. 3. A number of LDV models for a $0.1016 \mathrm{~m}$ (4 inch) pipe and $20 \%$ concentration.

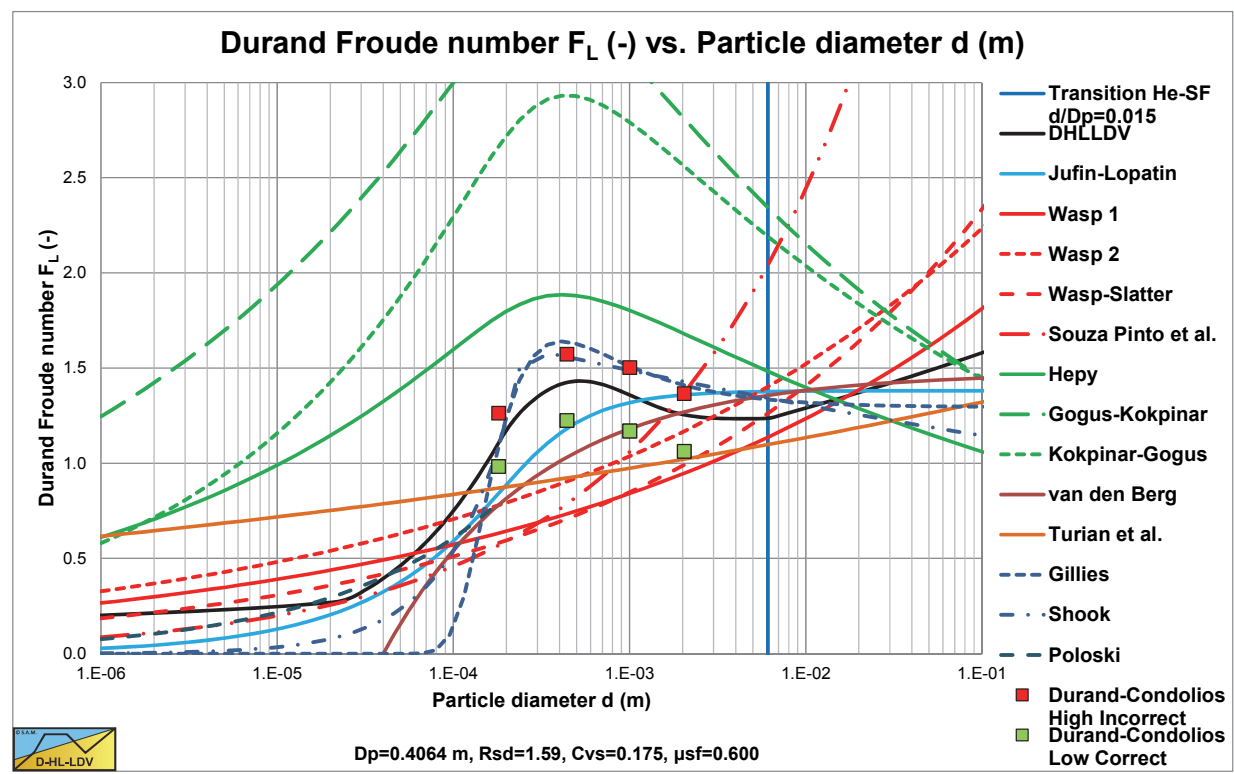

Fig. 4. A number of LDV models for a $0.4064 \mathrm{~m}$ (16 inch) pipe and $20 \%$ concentration. 
With a strong relation with the particle diameter and a weak relation for the pipe diameter, the pipe diameter will follow the particle diameter. Another reason may be the fact that they used pipe diameters up to $0.1524 \mathrm{~m}(6 \mathrm{inch})$ and the smaller the pipe diameter the more probable the occurrence of a sliding bed and other limiting conditions, due to the larger hydraulic gradient helping the bed to start sliding.

The figures show that for small pipe diameters all models are close. The reason is probably that most experiments are carried out with small pipe diameters. Only Jufin and Lopatin (1966) covered a range from $0.02 \mathrm{~m}$ to $0.9 \mathrm{~m}$ pipe diameters. Recently Thomas (2014) gave an overview and analysis of the LDV (or sometimes the LSDV). He repeated the findings that the LDV depends on the pipe diameter with a power smaller than 0.5 but larger than 0.1 . The value of 0.1 is for very small particles, while for normal sand and gravels a power is expected between $1 / 3$ according to Jufin and Lopatin (1966) and 1/2 according to Durand and Condolios (1952). Most equations are one term equations, making it impossible to cover all aspects of the LDV behavior. Only Gillies (1993) managed to construct an equation that gets close to the original Durand and Condolios (1952) graph.

In Fig. 3 and Fig. 4 the incorrect data of Durand and Condolios (1952) are the ones used in many textbooks. However Durand (1953) forgot to divide the $F_{L}$ value by the square root of the relative submerged density. Based on the original data the correct values are determined. The incorrect values however match later experiments better.

\section{CONCLUSIONS LITERATURE}

The models analyzed result in a number of dominating parameters. These are the particle diameter $d$, the pipe diameter $D_{p}$, the liquid density $\rho_{l}$ and kinematic viscosity $v_{l}$, the solids density $\rho_{s}$, the sliding friction coefficient $\mu_{s f}$, the bed concentration $C_{v b}$ and the spatial volumetric concentration $C_{v s}$. Derived parameters are the relative submerged density $R_{s d}$, the Darcy Weisbach friction factor for pure liquid flow $\lambda_{l}$ and the thickness of the viscous sub-layer $\delta_{v}$. Dimensionless numbers are not considered at first, since they may lead to wrong interpretations.

Lately Lahiri (2009) performed an analysis using artificial neural network and support vector regression. Azamathulla and Ahmad (2013) performed an analysis using adaptive neurofuzzy interference system and gene-expression programming. Although these methodologies may give good correlations, they do not explain the physics. Lahiri (2009) however did give statistical relations for the dependency on the volumetric concentration, the particle diameter, the pipe diameter and the relative submerged density.

Resuming, the following conclusions can be drawn for sand and gravel:

1. The pipe diameter $D_{p}$ : The LDV is proportional to the pipe diameter $D_{p}$ to a power between $1 / 3$ and $1 / 2$ (about 0.4 ) for small to large particles (Thomas (1979), Wasp et al. (1977), Lahiri (2009) and Jufin and Lopatin (1966)) and a power of about 0.1 for very small particles (Thomas (1979), Wilson and Judge (1976), Sanders et al. (2004) and Poloski et al. (2010)).

2. The particle diameter $d$ : The LDV has a lower limit for very small particles, after which it increases to a maximum at a particle diameter of about $d=0.5 \mathrm{~mm}$ (Thomas (1979), Thomas (2014), Durand and Condolios (1952), Gillies (1993) and Poloski et al. (2010)). For medium sized particles with a particle size $d>0.5 \mathrm{~mm}$, the $F_{L}$ value decreases to a minimum for a particle size of about $d=2 \mathrm{~mm}$ (Durand and Condolios, 1952;
Gillies, 1993; Poloski et al., 2010). Above $2 \mathrm{~mm}$, the $F_{L}$ value will remain constant according to Durand and Condolios (1952) and Gillies (1993). For particles with $d / D_{p}>0.015$, the Wilson et al. (1992) criterion for real suspension/saltation, the $F_{L}$ value increases again. This criterion is based on the ratio particle diameter to pipe diameter and will start at a large particle diameter with increasing pipe diameter. Yagi et al. (1972) reported many data points in this region showing an increasing $F_{L}$ value.

3. The relative submerged density $R_{s d}$ : The relation between the LDV and the relative submerged density is not very clear, however the data shown by Kokpinar and Gogus (2001) and the conclusions of Lahiri (2009) show that the $F_{L}$ value decreases with increasing solids density and thus relative submerged density $R_{s d}$ to a power of -0.2 to -0.4 .

4. The spatial volumetric concentration $C_{v s}$ : The volumetric concentration leading to the maximum LDV is somewhere between $15 \%$ and $20 \%$ according to Durand and Condolios (1952). Lahiri (2009) reported a maximum at about $17.5 \%$, while Poloski et al. (2010) derived $15 \%$. This maximum LDV results from on one hand a linear increase of the sedimentation with the concentration and on the other hand a reduced sedimentation due to the hindered setting. These two counteracting phenomena result in a maximum, which is also present in the equation of the potential energy. For small concentrations a minimum LDV is observed by Durand and Condolios (1952). This minimum LDV increases with the particle diameter and reaches the LDV of $20 \%$ at a particle diameter of $2 \mathrm{~mm}$ with a pipe diameter of $0.1524 \mathrm{~m}$ (6 inch).

For the dredging industry the Jufin and Lopatin (1966) equation gives a good approximation for sand and gravel, although a bit conservative. The model of Berg (1998) is suitable for large diameter pipes as used in dredging for sand and/or gravel, but underestimates the LDV for pipe diameters below $0.8 \mathrm{~m}$. Both models tend to underestimate the LDV for particle diameters below $1 \mathrm{~mm}$. The Jufin \& Lopatin equation is for sand and gravel:

$$
v_{l s, l d v}=8.3 \cdot\left(C_{v t} \cdot \psi^{*}\right)^{1 / 6} \cdot D_{p}^{1 / 3}
$$

or

$$
F_{L}=\frac{8.3 \cdot\left(C_{v t} \cdot \psi^{*}\right)^{1 / 6} \cdot D_{p}^{1 / 3}}{\sqrt{2 \cdot g \cdot R_{s d} \cdot D_{p}}}
$$

$$
\text { with : } \psi^{*}=\left(\frac{v_{t}}{\sqrt{g \cdot d}}\right)^{3 / 2}
$$

\section{STARTING POINTS DHLLDV FRAMEWORK}

Analyzing the literature, equations and experimental data, the LDV can be divided into 5 regions for sand and gravel, see Fig. 5:

1. Very small particles, smaller than about $50 \%$ of the thickness of the viscous sub layer, giving a lower limit of the LDV. This is for particles up to about $0.015 \mathrm{~mm}$ in large pipes to $0.04 \mathrm{~mm}$ in very small pipes.

2. Small particles up to about $0.2 \mathrm{~mm}$, a smooth bed, show an increasing LDV with increasing particle diameter. 
3. Medium particles with a diameter from $0.2 \mathrm{~mm}$ up to a diameter of $2 \mathrm{~mm}$, a transition zone from a smooth bed to a rough bed. First the LDV increases to a particle diameter of about $0.5 \mathrm{~mm}$, after which it decreases slowly to an asymptotic value at a diameter of about $2 \mathrm{~mm}$.

4. Large particles with a diameter larger than $2 \mathrm{~mm}$, a rough bed, giving a constant LDV.

5. Particles with a particle diameter to pipe diameter ratio larger than about 0.015 cannot be carried by turbulent eddies, just because eddies are not large enough. This will probably result in an increasing LDV with the particle diameter. Yagi et al. (1972) reported many data points in this region showing this increase.

The above conclusions are the starting points of the DHLLDV Limit Deposit Velocity Model and has been shown in the figures. Fig. 5 shows the flow chart for the determination of the LDV, based on these findings.

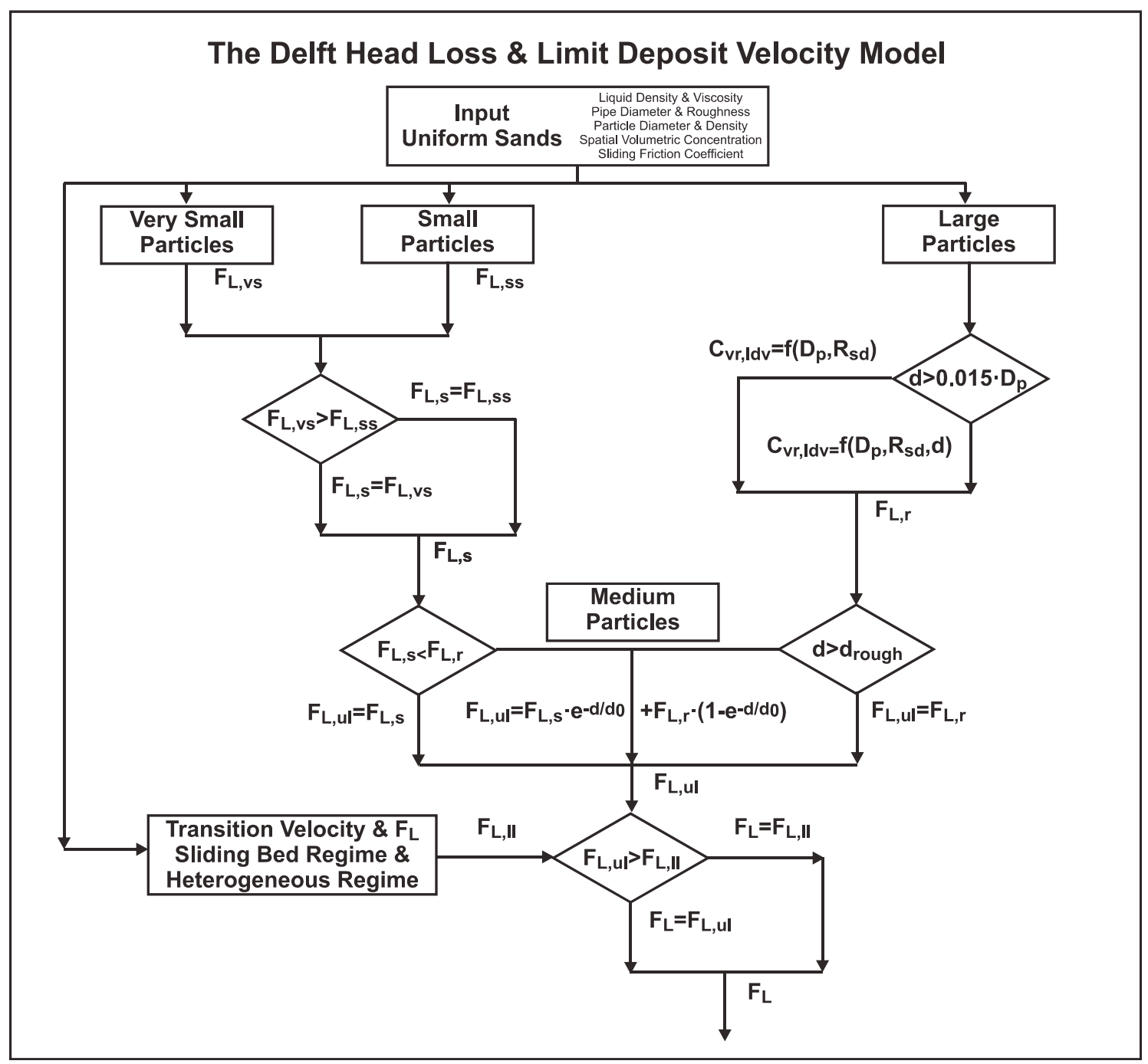

Fig. 5. The flow chart for the determination of the LDV.

\section{REGION 1: VERY SMALL PARTICLES, THE LOWER LIMIT}

Thomas (1979) investigated the Limit Deposit Velocity for very small particles and found that there is a lower limit to the LDV independent of the particle size. This lower limit is based on the assumption that the very small particles are smaller than the thickness of the viscous sub layer and still settle in this laminar flow, while particles in the turbulent flow are carried by the small eddies. At the moment of sliding the shear stress $\tau_{12}$ at the top of the thin layer with thickness $h$, equals the sliding friction force divided by the total sliding surface:

$$
\begin{aligned}
\tau_{12} & =\frac{F_{s f}}{D_{p} \cdot \Delta L}=\mu_{s f} \cdot h \cdot \rho_{l} \cdot R_{s d} \cdot(1-n) \cdot g \\
& =\mu_{s f} \cdot h \cdot \rho_{l} \cdot R_{s d} \cdot C_{v b} \cdot g
\end{aligned}
$$

The thickness of the thin layer is assumed to be equal to the thickness of the viscous sub layer: 


$$
h=\delta_{v}=11.6 \cdot \frac{v_{l}}{u_{*}}
$$

Thomas (1979) used a factor 5 instead of 11.6. Substituting this layer thickness gives:

$$
\begin{aligned}
\tau_{12} & =11.6 \cdot \mu_{s f} \cdot \frac{v_{l}}{u_{*}} \cdot \rho_{l} \cdot R_{s d} \cdot C_{v b} \cdot g \\
& =\rho_{l} \cdot u_{*}^{2}
\end{aligned}
$$

This gives for the friction velocity:

$$
u_{*}^{3}=11.6 \cdot \mu_{s f} \cdot v_{l} \cdot R_{s d} \cdot C_{v b} \cdot g
$$

or

$u_{*}=2.26 \cdot\left(\mu_{s f} \cdot v_{l} \cdot R_{s d} \cdot C_{v b} \cdot g\right)^{1 / 3}$

With a bed concentration $C_{v b}=0.6$ and a sliding friction coefficient of $\mu_{s f}=0.4$ this gives (Thomas (1979) found 1.1 instead of 1.4):

$$
u_{*}=1.4 \cdot\left(v_{l} \cdot R_{s d} \cdot g\right)^{1 / 3}
$$

or

$u_{*}=0.0387 \mathrm{~m} / \mathrm{s}$

For sand with a relative submerged density of $R_{s d}=1.65$, water with a viscosity of $v_{l}=0.0000013 \mathrm{~m}^{2} / \mathrm{s}$ this gives a friction velocity of $0.0387 \mathrm{~m} / \mathrm{s}$. In terms of the Limit Deposit Velocity the following is found:

$$
\begin{aligned}
& u_{*}=\sqrt{\frac{\lambda_{l}}{8}} \cdot v_{l s, l d v} \\
& =2.26 \cdot\left(\mu_{s f} \cdot v_{l} \cdot R_{s d} \cdot C_{v b} \cdot g\right)^{1 / 3} \\
& v_{l s, l d v}=2.26 \cdot\left(\mu_{s f} \cdot v_{l} \cdot R_{s d} \cdot C_{v b} \cdot g\right)^{1 / 3} \cdot \sqrt{\frac{8}{\lambda_{l}}} \\
& \Rightarrow \quad v_{l s, l d v}=3.96 \cdot \frac{\left(v_{l} \cdot R_{s d} \cdot g\right)^{1 / 3}}{\sqrt{\lambda_{l}}}
\end{aligned}
$$

For small pipes with $\lambda_{l}=0.03$ this gives $v_{l s, l d v}=0.64 \mathrm{~m} / \mathrm{s}$, for medium pipes with $\lambda_{1}=0.02$ this gives $v_{l s, l d v}=0.78 \mathrm{~m} / \mathrm{s}$ and for large pipe diameters with $\lambda_{1}=0.01$ this gives $v_{l s, l d v}=1.10 \mathrm{~m} / \mathrm{s}$. The LDV increases with the pipe diameter to a power of about 0.1 as already noted by Thomas (1979). Because Thomas (1979) used a factor 5 for the thickness of the viscous sub layer instead of the 11.6 as used here, he found a theoretical coefficient of 0.933 instead of the coefficient 1.4 found here. Based on his experiments he corrected this factor to 1.1. Now the value of the thickness of the viscous sub layer is just a mathematical thickness, so it is very well possible that the thickness as used here is smaller than the one using the coefficient of 11.6 and larger than using the coefficient of 5 . Based on the experimental coefficient of 1.1 as found by Thomas (1979) the coefficient for the thickness of the viscous sub layer involved should be 5.62 , almost $50 \%$ of the thickness of the viscous sub layer. It should be noted that the velocity found this way is in fact the LSDV and not the LDV. The LSDV is the Limit of Stationary Deposit, so the line speed where the bed starts sliding, while the LDV is the Limit Deposit Velocity, the line speed where all particles are in suspension and there is also no sliding bed anymore. The LDV is always higher or equal than the LSDV. However it is the question whether for very small particles residing in the viscous sub layer, the situation of having $100 \%$ of the particles in suspension will ever occur. Another issue is the apparent viscosity. As the volumetric concentration increases, so does the apparent viscosity for very small particles, according to Thomas (1965). To include the viscosity effect and to be on the safe side, the factor 1.4 is used in the DHLLDV framework. In terms of the Durand \& Condolios Froude number this gives:

$$
\begin{aligned}
& F_{L}=\frac{v_{l s, l d v}}{\sqrt{2 \cdot g \cdot R_{s d} \cdot D_{p}}} \\
& =2.26 \cdot \frac{\left(\mu_{s f} \cdot v_{l} \cdot R_{s d} \cdot C_{v b} \cdot g\right)^{1 / 3}}{\sqrt{2 \cdot g \cdot R_{s d} \cdot D_{p}}} \cdot \sqrt{\frac{8}{\lambda_{l}}}
\end{aligned}
$$

Sanders et al. (2004) modified the Thomas (1979) equation by adding the effect of the particle diameter. Their equation is validated in pipes of $0.103 \mathrm{~m}\left(d_{50}=0.088 \mathrm{~mm}\right)$ and $0.265 \mathrm{~m}$ $\left(d_{50}=0.169 \mathrm{~mm}\right)$ pipe diameter. The equation as published is implicit, but with some mathematics it can be made explicit, giving:

$$
\begin{aligned}
v_{l s, l d v}= & 2.26 \cdot A \cdot \sqrt{\frac{8}{\lambda_{l}}} \\
& \cdot \frac{0.77}{\left(\left(C_{v b}-C_{v s}\right)^{0.88}\right)^{1 / 3}-0.25 \cdot \frac{d}{v_{l}} \cdot A}
\end{aligned}
$$

with: $A=\left(\mu_{s f} \cdot v_{l} \cdot R_{s d} \cdot C_{v b} \cdot g\right)^{1 / 3}$

So the addition is a multiplication factor which equals unity for very small particles and a concentration of $17.5 \%$. The multiplication factor increases with increasing particle diameter and increasing spatial volumetric concentration. It is however a question whether the denominator of this factor should only contain the particle diameter $d$ or also the pipe diameter $D_{p}$, since the experiments also used an increased particle diameter and pipe diameter together.

\section{REGION 2: SMOOTH BED, SMALL PARTICLES}

When the particles do not fit in the viscous sub-layer anymore, there is another mechanism. Small particles are kept in suspension by the turbulent eddies if there is enough turbulent energy in the liquid flow. This will of course only be valid for particles small enough to be carried by these eddies. The poten- 
tial energy losses of the particles are decreasing with increasing line speed, Miedema and Ramsdell (2013), while the energy losses of the liquid flow are increasing with increasing line speed. Now it is assumed that a certain fraction of the energy losses of the liquid flow is available for the suspension of the particles. This fraction is defined as $1 / \alpha_{p}{ }^{3}$. So at the line speed where the potential energy losses are equal to $1 / \alpha_{p}{ }^{3}$ times the liquid energy losses, all particles will be in suspension. At a lower line speed some particles will form a bed at the bottom of the pipe. The potential energy losses of the particles can be expressed by means of the excess hydraulic gradient due to potential energy, Miedema and Ramsdell (2013). The liquid flow energy losses can be expressed in terms of the liquid hydraulic gradient. This gives at the LDV:

$$
\frac{v_{t} \cdot\left(1-\frac{C_{v s}}{\kappa_{C}}\right)^{\beta} \cdot C_{v s} \cdot R_{s d}}{v_{l s, l d v}}=\frac{1}{\alpha_{p}^{3}} \cdot \frac{\lambda_{l} \cdot v_{l s, l d v}^{2}}{2 \cdot g \cdot D_{p}}
$$

Solving for the Limit Deposit Velocity gives:

$$
v_{l s, l d v}^{3}=\alpha_{p}^{3} \cdot \frac{v_{t} \cdot\left(1-\frac{C_{v s}}{\kappa_{C}}\right)^{\beta} \cdot C_{v s} \cdot\left(2 \cdot g \cdot R_{s d} \cdot D_{p}\right)}{\lambda_{l}}
$$

This equation shows that the Limit Deposit Velocity of small particles depends on the terminal settling velocity with a correction for hindered settling $v_{t} \cdot\left(1-C_{v s} / \kappa_{C}\right)^{\beta}$, the spatial volumetric concentration $C_{v s}$, the relative submerged density $R_{s d}$, the pipe diameter $D_{p}$ and the Darcy Weisbach friction factor $\lambda_{l}$. The term $\left(1-C_{v s} / \kappa_{C}\right)^{\beta} \cdot C_{v s}$ should have a maximum at a spatial concentration of about $17.5 \%$ independ on the value of the power $\beta$ and the factor $\kappa_{C}$. In terms of the Durand \& Condolios LDV Froude number $F_{L}$ factor this can be written as:

$$
\begin{aligned}
& F_{L, s}= \frac{v_{l s, l d v}}{\left(2 \cdot g \cdot R_{s d} \cdot D_{p}\right)^{1 / 2}} \\
&= \alpha_{p} \cdot\left(\frac{v_{t} \cdot\left(1-\frac{C_{v s}}{\kappa_{C}}\right)^{\beta} \cdot C_{v s}}{\lambda_{l} \cdot\left(2 \cdot g \cdot R_{s d} \cdot D_{p}\right)^{1 / 2}}\right)^{1 / 3} \\
& \text { with : } \quad \alpha_{p}=3.5 \cdot\left(\frac{1.65}{R_{s d}}\right)^{1 / 9} \\
& \kappa_{C}=0.175 \cdot(1+\beta)
\end{aligned}
$$

The values found for $\alpha_{p}=3.5$ and $\kappa_{C}$ are based on many experiments from literature. The value of $\alpha_{p}$ of 3.5 found shows that the LDV occurs when the potential energy losses of the particles are about $2.33 \%$ of the energy losses of the liquid flow for small particles. The value of $\kappa_{C}$ means that the average particle is at $\kappa_{C}$ of the radius of the pipe, measured from the bottom of the pipe and not at the radius. This is in fact the concentration eccentricity factor. It also appears that the Limit Deposit Velocity found for small particles is close to the transition between the heterogeneous regime and the homogeneous regime, which makes sense, because in the homogeneous regime all particles are supposed to be in suspension.

The value of $\alpha_{p}=3.5$ is based on the maximum LDV values found in literature and not on a best fit. In fact $\alpha_{p}$ may vary from 3.0 as a lower limit to 3.5 as an upper limit. So the value of 3.5 is conservative. A best fit would probably give a value of about 3.25. However in dredging the LDV should be a safe LDV, since the particle size, the concentration and the line speed may vary in time.

\section{REGION 4: ROUGH BED, LARGE PARTICLES}

When the particles are larger, the small eddies are not strong enough to keep them in suspension. The transport mechanism over the bottom of the pipe will be more saltation or similar to sheet flow. For medium and large particles in sedimentation transport it is often assumed that particles are in suspension or in saltation when the friction velocity is larger than the terminal settling velocity, $u_{*}>v_{t}$. Larger particles will be more difficult to keep in suspension, but at a certain bed shear stress saltation will take over. Whether this occurs exactly at $u_{*}=v_{t}$ can be questioned, but some proportionality seems reasonable since the friction velocity is proportional to the bed shear stress. The Shields-Parker diagram, (Garcia, 2008), uses this assumption. Substituting the friction velocity for the terminal settling velocity gives:

$$
\frac{u_{*} \cdot\left(1-\frac{C_{v s}}{\kappa_{C}}\right)^{\beta} \cdot C_{v s} \cdot R_{s d}}{v_{l s, l d v}}=\frac{1}{\alpha_{p}^{3}} \cdot \frac{\lambda_{l} \cdot v_{l s, l d v}^{2}}{2 \cdot g \cdot D_{p}}
$$

The question is now, what to use for the friction velocity $u *$ ? In general a limiting volumetric bed concentration can be assumed for a line speed just below the Limit Deposit Velocity $C_{v s, l d v}$. This limiting concentration is the cross section of particles in the bed at the LDV divided by the pipe cross section, and not the total concentration, since most of the particles are in suspension or saltation.

The limiting volumetric bed concentration can be divided by the bed concentration $C_{v b}$ giving a relative limiting volumetric bed concentration $C_{v r, l d v}$. This relative limiting volumetric bed concentration gives the fraction of the bed related to the pipe cross section. In terms of the bed shear stress this gives:

$u *=\sqrt{\frac{\tau_{12}}{\rho_{l}}}$

$\tau_{12}=\mu_{s f} \cdot g \cdot \rho_{l} \cdot R_{s d} \cdot \frac{\pi}{4} \cdot D_{p} \cdot C_{v r, l d v} \cdot C_{v b}$

The shear stress can be derived by assuming that there is a thin layer of sand covering the bottom of the pipe with a thickness $h$ over the full diameter of the pipe. The weight of this thin layer is:

$F_{W}=h \cdot D_{p} \cdot \Delta L \cdot \rho_{l} \cdot R_{s d} \cdot(1-n) \cdot g$

The sliding friction force of this thin layer of sand on the bottom of the pipe is: 
$F_{s f}=\mu_{s f} \cdot h \cdot D_{p} \cdot \Delta L \cdot \rho_{l} \cdot R_{s d} \cdot(1-n) \cdot g$

At the moment of sliding the shear stress at the top of the thin layer equals the sliding friction force divided by the total sliding surface:

$\tau_{12}=\frac{F_{s f}}{D_{p} \cdot \Delta L}=\mu_{s f} \cdot h \cdot \rho_{l} \cdot R_{s d} \cdot(1-n) \cdot g$

The volumetric concentration of the bed with respect to the full cross section of the pipe is:

$C_{v s, l d v}=\frac{h \cdot D_{p} \cdot(1-n)}{\frac{\pi}{4} \cdot D_{p}^{2}}=\frac{4}{\pi} \cdot \frac{h \cdot(1-n)}{D_{p}}$

This gives for the thickness of the thin layer $h$ :

$$
h=C_{v S, l d v} \cdot \frac{\pi}{4} \cdot \frac{D_{p}}{(1-n)}
$$

So now the shear stress equals, in terms of the relative limiting volumetric concentration:

$$
\begin{aligned}
\tau_{12} & =\mu_{s f} \cdot C_{v s, l d v} \cdot \frac{\pi}{8} \cdot \rho_{l} \cdot 2 \cdot g \cdot R_{s d} \cdot D_{p} \\
& =\mu_{s f} \cdot \frac{\pi}{8} \cdot \rho_{l} \cdot\left(2 \cdot g \cdot R_{s d} \cdot D_{p}\right) \cdot \frac{C_{v s, l d v}}{C_{v b}} \cdot C_{v b} \\
\tau_{12} & =\mu_{s f} \cdot \frac{\pi}{8} \cdot \rho_{l} \cdot\left(2 \cdot g \cdot R_{s d} \cdot D_{p}\right) \cdot C_{v r, l d v} \cdot C_{v b}
\end{aligned}
$$

Substituting the shear stress for the friction velocity gives:

$$
\frac{\sqrt{\frac{\tau_{12}}{\rho_{l}}} \cdot\left(1-\frac{C_{v s}}{\kappa_{C}}\right)^{\beta} \cdot C_{v s} \cdot R_{s d}}{v_{l s, l d v}}=\frac{1}{\alpha_{p}^{3}} \cdot \frac{\lambda_{l} \cdot v_{l s, l d v}^{2}}{2 \cdot g \cdot D_{p}}
$$

Thus, based on the limiting volumetric concentration:

$$
\begin{array}{r}
\frac{\sqrt{\frac{\rho_{l} \cdot \mu_{s f} \cdot \frac{\pi}{8} \cdot C_{v r, l d v} \cdot C_{v b} \cdot\left(2 \cdot g \cdot R_{s d} \cdot D_{p}\right)}{\rho_{l}}}}{v_{l s, l d v}} \\
. \frac{\left(1-\frac{C_{v s}}{\kappa_{C}}\right)^{\beta} \cdot C_{v s} \cdot R_{s d}}{v_{l s, l d v}}=\frac{1}{\alpha_{p}^{3}} \cdot \frac{\lambda_{l} \cdot v_{l s, l d v}^{2}}{2 \cdot g \cdot D_{p}}
\end{array}
$$

This can be rewritten as:

$$
\begin{aligned}
& \frac{\left(2 \cdot g \cdot R_{s d} \cdot D_{p}\right)^{1 / 2} \cdot\left(\mu_{s f} \cdot C_{v b} \cdot \frac{\pi}{8}\right)^{1 / 2}}{v_{l s, l d v}} \\
& \quad \frac{C_{v r, l d v}^{1 / 2} \cdot\left(1-\frac{C_{v s}}{\kappa_{C}}\right)^{\beta} \cdot C_{v s} \cdot R_{s d}}{v_{l s, l d v}}=\frac{1}{\alpha_{p}^{3}} \cdot \frac{\lambda_{l} \cdot v_{l s, l d v}^{2}}{2 \cdot g \cdot D_{p}}
\end{aligned}
$$

So the limit deposit velocity LDV is now for medium and large particles:

$$
\begin{aligned}
v_{l s, l d v}^{3}= & \alpha_{p}^{3} \cdot \frac{\left(1-\frac{C_{v s}}{\kappa_{C}}\right)^{\beta} \cdot C_{v s}}{\lambda_{l}} \\
& \cdot \frac{\left(\mu_{s f} \cdot C_{v b} \cdot \frac{\pi}{8}\right)^{1 / 2} \cdot C_{v r, l d v}^{1 / 2}}{\lambda_{l}} \\
& \cdot\left(2 \cdot g \cdot R_{s d} \cdot D_{p}\right)^{3 / 2}
\end{aligned}
$$

And the Durand \& Condolios LDV Froude number:

$$
F_{L, r}=\frac{v_{l s, l d v}}{\left(2 \cdot g \cdot R_{s d} \cdot D_{p}\right)^{1 / 2}}
$$

$$
=\alpha_{p} \cdot\left(\begin{array}{l}
\frac{\left(1-\frac{C_{v s}}{\kappa_{C}}\right)^{\beta} \cdot C_{v s}}{\lambda_{l}} \\
\cdot \frac{\left(\mu_{s f} \cdot C_{v b} \cdot \frac{\pi}{8}\right)^{1 / 2} \cdot C_{v r, l d v}^{1 / 2}}{\lambda_{l}}
\end{array}\right)^{1 / 3}
$$

The limiting relative volumetric concentration $C_{v r, l d v}$ appears to depend on the pipe diameter $D_{p}$ and the relative submerged density $R_{s d}$. With a constant thickness of the thin layer $h$, the amount of solids in this thin layer is proportional to the pipe diameter $D_{p}$. The cross section of the pipe however is proportional to the pipe diameter $D_{p}$ squared. So the limiting relative volumetric concentration $C_{v r, l d v}$ will be reversely proportional to the pipe diameter. The limiting relative volumetric concentration $C_{v r, l d v}$ is decreasing with increasing relative submerged density $R_{s d}$. This can be explained by assuming that a certain critical bed shear stress $\tau_{12}$ requires a decreasing relative volumetric concentration $C_{v r, l d v}$ with an increasing relative submerged density $R_{s d}$, giving: 


$$
\begin{aligned}
C_{v r, l d v} & =0.00004 \cdot D_{p}^{-1} \cdot\left(\frac{R_{s d}}{1.65}\right)^{-1} \\
& =\frac{0.0013}{2 \cdot g \cdot R_{s d} \cdot D_{p}}
\end{aligned}
$$

\section{REGION 5: SLIDING FLOW, VERY LARGE PARTICLES}

When the particles are very large compared to the pipe diameter, the particles will not be suspended or have saltating behavior, but will flow over the bottom of the pipe. So the transition of a sliding bed to heterogeneous flow will not or just partly be present. Physically this can be explained in the following way: At low line speeds a sliding bed will occur with a bed concentration of $C_{v b}$. Because of the high bed shear stress to top of the bed will behave as sheet flow, moving faster than the bottom of the bed. When the line speed increases, the bed concentration will decrease (Gillies, 1993) as is implemented in the SRC model. At a certain line speed the bed concentration reaches a value where the particles do not touch each other anymore if the bed would be in rest. However, due to the particle-pipe wall and particle-particle interactions, the particles are still capable of transmitting the gravity forces, resulting in sliding friction with the pipe wall. One cannot speak of a sliding bed anymore, although a black box approach still shows sliding friction behavior. That's why this regime is named the sliding flow regime. One cannot really speak of an LDV here, but a value is required for the determination of the slip ratio as part of the DHLLDV framework. The experiments of Yagi et al. (1972) show this behavior.

In the sliding flow regime, the particles are so large that they flow over the bottom of the pipe giving sliding friction behavior, the limiting relative volumetric concentration increases with an increasing particle diameter to pipe diameter ratio. The criterion for fully stratified flow of Wilson et al. (1992) is applied here. When the particles are large it is also the question whether they still fit in the thin layer with thickness $h$.

$$
\begin{aligned}
d \leq 0.015 \cdot D_{p} \\
C_{v r, l d v}=0.00004 \cdot D_{p}^{-1} \cdot\left(\frac{R_{s d}}{1.65}\right)^{-1} \\
=\frac{0.0013}{2 \cdot g \cdot R_{s d} \cdot D_{p}}
\end{aligned}
$$

$$
\begin{aligned}
d>0.015 \cdot D_{p} \\
C_{v r, l d v}=0.00004 \cdot D_{p}^{-1} \cdot\left(\frac{R_{s d}}{1.65}\right)^{-1} \cdot\left(\frac{d}{0.015 \cdot D_{p}}\right)^{1 / 2} \\
=\frac{0.0106}{2 \cdot g \cdot R_{s d} \cdot D_{p}} \cdot\left(\frac{d}{D_{p}}\right)^{1 / 2}
\end{aligned}
$$

\section{REGION 3: THE TRANSITION REGION, MEDIUM PARTICLES}

The transition from a smooth bed with small particles to a rough bed with large particles requires some special attention. This transition can be compared to the transition from a hydraulic smooth bed to a hydraulic rough bed, considering the starting elevation of the logarithmic velocity profile, the Law of the Wall (Garcia, 2008). There will be a combination of the turbulent mechanism for small particles and the shear stress mechanism for large particles. Following the shape of the LDV curve of Durand and Condolios (1952) and Gillies (1993) there should be a maximum LDV for particles with a diameter close to $0.5 \mathrm{~mm}$.

The $F_{L}$ value in the transition region can now be determined according to:

$$
\begin{aligned}
& F_{L, s} \leq F_{L, r} \quad \Rightarrow \quad F_{L}=F_{L, s} \\
& F_{L, s}>F_{L, r} \Rightarrow \\
& F_{L}=F_{L, s} \cdot e^{-d / d_{0}}+F_{L, r} \cdot\left(1-e^{-d / d_{0}}\right) \\
& \text { with : } \quad d_{0}=0.0005 \cdot\left(\frac{1.65}{R_{s d}}\right)^{1 / 2}
\end{aligned}
$$

The coefficient $d_{0}$ is found by calibrating the equations on as much experimental data as possible. It is not tried to get the best fit, but to be on the safe side, $90-95 \%$ of the data points should be below the resulting LDV curve. In general, the maximum LDV curves are found at a concentration of about $17.5 \%$. Above $17.5 \%$ the LDV decreases again.

It is remarkable that the LDV shows a maximum for sand particles with a diameter of $0.5 \mathrm{~mm}$, while the Shields curve (Miedema, 2012a) and (Miedema, 2012b) shows a minimum for about the same particle diameter. The curves are mirrored around a horizontal axis. The difference between the LDV curve and the Shields curve is however, the Shields curve deals with the initiation of motion, while the LDV curve deals with suspension, saltation or sliding of the bed.

\section{THE TRANSITION SLIDING BED - HETEROGENEOUS, A LOWER LIMIT}

When the Limit Deposit Velocity found this way is smaller than the transition velocity between the sliding bed regime and the heterogeneous regime, the latter should be chosen for the LDV. Whether this is exactly this transition velocity is a question, but since a LDV smaller than this transition velocity would result in an $E_{r h g}$ value in the heterogeneous regime higher than the sliding bed $E_{r h g}$, which is impossible, this transition is chosen as a limitation to the LDV curves.

When a sliding bed is present, particles will be in suspension above the sliding bed. The higher the line speed, the more particles will be in suspension. The interaction between the particles in suspension and the particles in the bed will still be by inter particle interactions, reason that the sliding bed is still carrying the weight of all the particles in suspension. Apparently the weight of all the particles is resulting in sliding friction. At a certain line speed all the particles will be in suspension or saltating and the sliding bed regime transits to heterogeneous flow. The particles now interact with the pipe wall by collisions and 
not by sliding friction anymore. This transition will, in reality, not be too well defined. However in this model for now it is assumed to be a sharp transition. At the Limit Deposit Velocity the excess pressure losses of both regimes should be equal, Miedema (2015c), giving:

$$
\begin{aligned}
\mu_{s f}= & \frac{v_{t}}{v_{l s, l d v}} \cdot\left(1-\frac{C_{v_{S}}}{\kappa_{C}}\right)^{\beta} \\
& +\frac{7.5^{2}}{\lambda_{l}} \cdot\left(\frac{1}{\sqrt{C_{x}}}\right)^{8 / 3} \cdot\left(\frac{\left(v_{l} \cdot g\right)^{1 / 3}}{v_{l s, l d v}}\right)^{2}
\end{aligned}
$$

This equation shows that the transition between the sliding bed regime and the heterogeneous regime depends on the sliding friction coefficient. Implicitly Newitt et al. (1955) already found this, but didn't explicitly mention this, because they assumed that potential energy is responsible for all the excess head losses in heterogeneous flow. The equation derived is a second degree function and can be written as:

$$
\begin{gathered}
-v_{l s, l d v}^{2}+\frac{v_{t} \cdot\left(1-\frac{C_{v s}}{\kappa_{C}}\right)^{\beta}}{\mu_{s f}} \cdot v_{l s, l d v} \\
+\frac{\frac{7.5^{2}}{\lambda_{l}} \cdot\left(\frac{1}{\sqrt{C_{x}}}\right)^{8 / 3} \cdot\left(v_{l} \cdot g\right)^{2 / 3}}{\mu_{s f}}=0
\end{gathered}
$$

with:

$$
\begin{aligned}
& A=-1 \\
& B=\frac{v_{t} \cdot\left(1-\frac{C_{v s}}{\kappa_{C}}\right)^{\beta}}{\mu_{s f}} \\
& C=\frac{\frac{7.5^{2}}{\lambda_{l}} \cdot\left(\frac{1}{\sqrt{C_{x}}}\right)^{8 / 3} \cdot\left(v_{l} \cdot g\right)^{2 / 3}}{\mu_{s f}} \\
& v_{l s, l d v}=\frac{-B-\sqrt{B^{2}-4 \cdot A \cdot C}}{2 \cdot A}
\end{aligned}
$$

\section{THE RESULTING LIMIT DEPOSIT VELOCITY CURVES}

In slurry transport applications like dredging, the slurry transport process is not steady state, but dynamical. At the entrance of the pipeline the volumetric concentration and the particle size distribution, PSD, will vary in time. Because of this the hydraulic gradient will vary, resulting in a varying line speed. Even with steady state volumetric concentration and PSD, moving dunes and large vortices may occur. So to be safe, the maximum LDV curve should be used, which occurs at a volumetric concentration of about $17.5 \%$.

Fig. 6 shows the construction of the LDV curve for sands and gravels in a 6 inch $(0.1524 \mathrm{~m})$ pipe, for a $17.5 \%$ spatial volumetric concentration. Up to a particle diameter of about $0.2 \mathrm{~mm}$ the resulting curve follows the smooth curve (red line). From $0.2 \mathrm{~mm}$ to about $2 \mathrm{~mm}$ the resulting curve follows the transition equation. Above $2 \mathrm{~mm}$, in this case the transition velocity between the sliding bed regime and the heterogeneous regime is followed. Finally above about $10 \mathrm{~mm}$ the sliding flow criteria is followed. The shape of the curve depends strongly on the pipe diameter. With small diameter pipes, the transition velocity between the sliding bed regime and the heterogeneous regime will be dominant for larger particles. While for large pipe diameters as used in dredging, this will hardly have any influence. The graph also contains the Jufin and Lopatin (1966) LDV curve, which is a one equation curve. This equation is chosen because of the large range of pipe diameters, from about 1 inch to $90 \mathrm{~cm}$, investigated. Of course a one equation model can never result in a complex curve based on different criteria as is shown here. However for practical purposes it also gives a good and safe result.

Fig. 7 shows the resulting LDV curves for volumetric concentrations from $1 \%$ to $25 \%$ on a linear horizontal axis. The horizontal axis is limited to particles of $2.5 \mathrm{~mm}$ in order to compare this graph with the famous Durand and Condolios (1952) graph. Although the Durand \& Condolios graph as copied by many authors contains an error on the vertical axis by a factor 1.285 , giving an asymptotic value of 1.34 for large particles (which should have been 1.05), and the resemblance with their graph is remarkable. Based on the experiments published by Gibert (1960) and others, the wrong Durand \& Condolios graph seems to be the right graph.

Fig. 8 shows the path of the LDV curve in a $E_{r h g}$ graph, the relative excess hydraulic gradient: $E_{r h g}=\left(i_{m}-i_{l}\right) /\left(R_{s d} \cdot C_{v s}\right)$.

The points are calculated for particle diameters of $0.1,0.2$, $0.3,0.5,0.75,1,3$ and $10 \mathrm{~mm}$. It is shown that for very small particles the LDV follows the homogeneous curve closely. From 0.2 to $3 \mathrm{~mm}$ the curve transits from the homogeneous curve to the sliding bed curve. Above $3 \mathrm{~mm}$ the LDV will follow the sliding bed curve. For smaller pipe diameters the LDV curve moves to the right, meaning that the distance between the homogeneous curve and the sliding bed curve decreases. Particles smaller than $3 \mathrm{~mm}$ will already reach the sliding bed curve. For larger pipe diameters the LDV curve moves to the left, meaning that the distance between the homogeneous curve and the sliding bed curve increases. Particles larger than $3 \mathrm{~mm}$ will not reach the sliding bed curve.

Fig. 9 shows the LDV as a function of the pipe diameter for 5 different particle sizes. In the graph also the average powers of the LDV curves with respect to the pipe diameters are shown. Equations (12) and (26) give a power of $1 / 3$ based on the presence of the pipe diameter in the equations. However, the equations also contain the Darcy-Weisbach friction factor in the denominator, increasing the powers slightly to a value around 0.4 . For small diameter pipes, the limiting transition velocity between the sliding bed and the heterogeneous regime results in a flat part. For particles with $d>0.015 \cdot D_{p}$, the adjustment of the LDV according to equation (28) will reduce the power. Based on this analysis, it is impossible to model the LDV with a one term equation. There are 4 different regions of the LDV and a limiting transition velocity. A smooth bed, a rough bed, a transition region and the criterion $d>0.015 \cdot D_{p}$ together with the limiting transition velocity between the sliding bed and the heterogeneous regime. Curve fit techniques on experimental data 


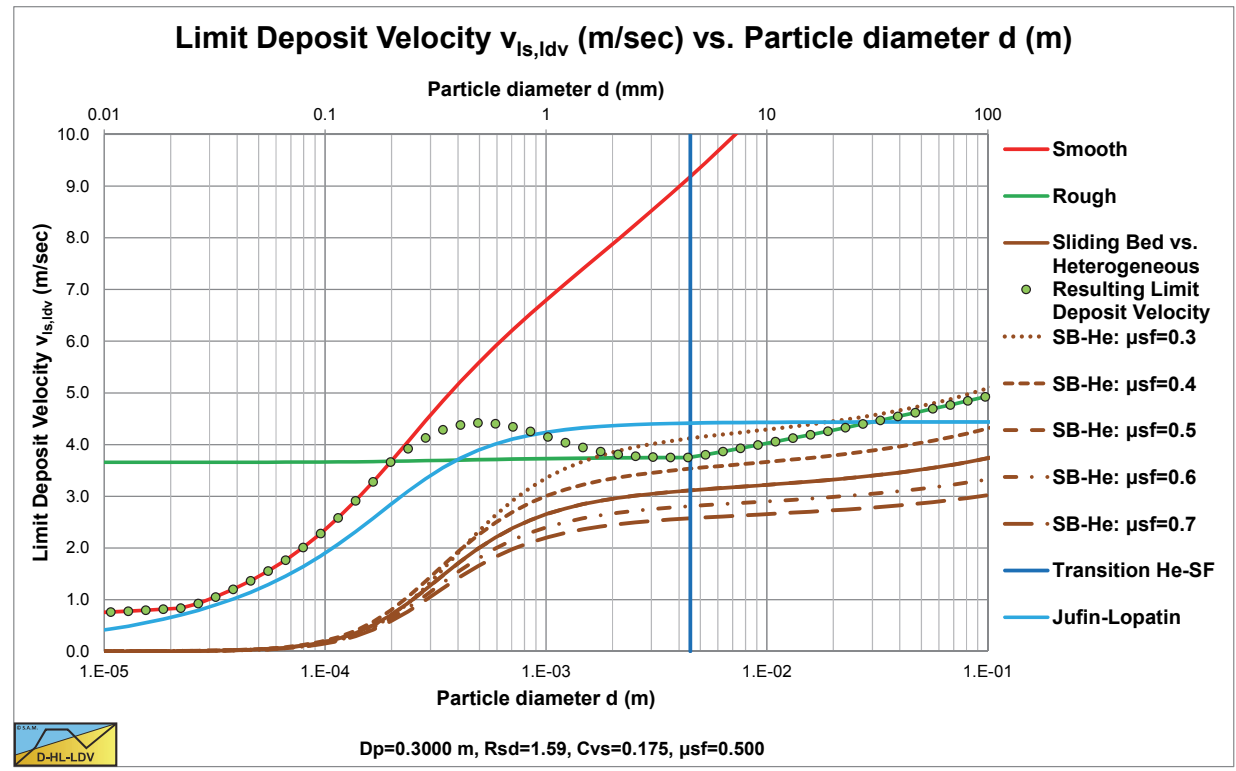

Fig. 6. The construction of the LDV curve.

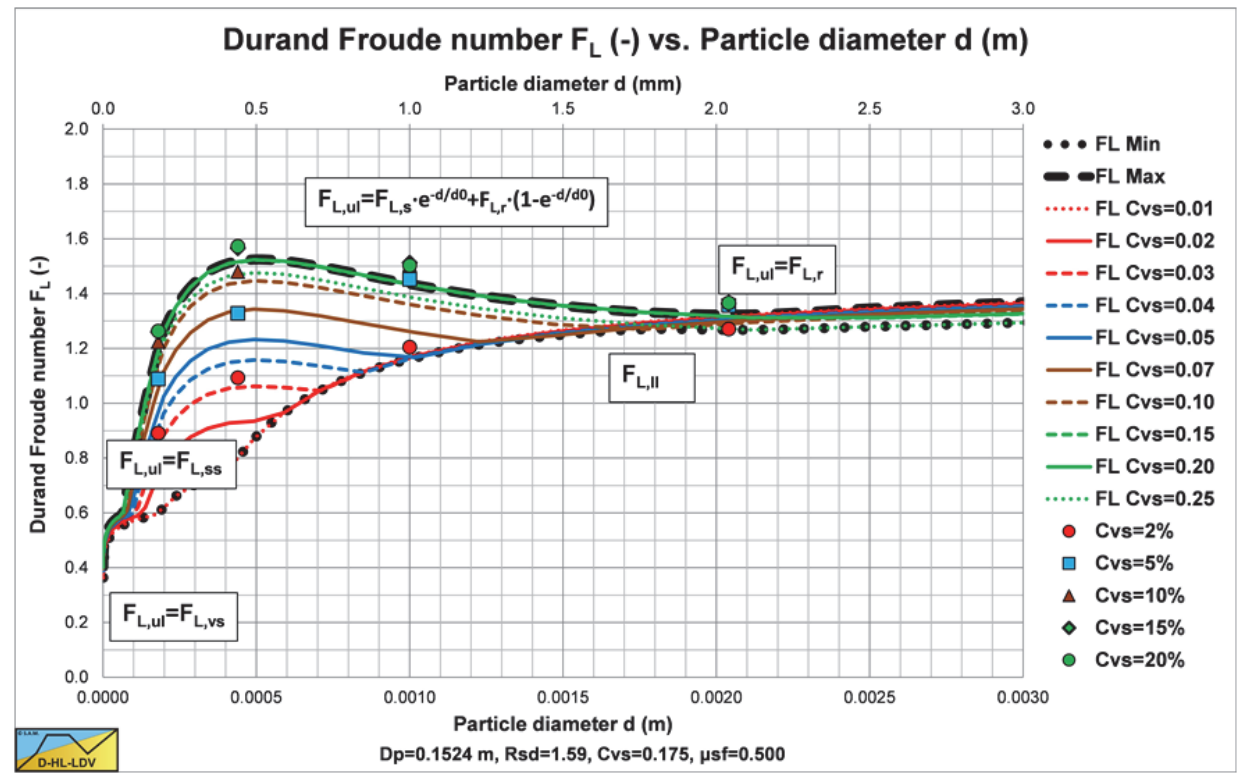

Fig. 7. The resulting LDV curve for different concentrations on a linear axis.

will always give a result, but this will be a different result based on the particle and pipe diameters used in the experiments.

\section{CONCLUSIONS \& DISCUSSION}

There are many definitions and names for the critical velocity. Here the critical velocity is defined as the Limit Deposit Velocity, the line speed above which there is no stationary bed or sliding bed. Below the LDV there may be either a stationary or fixed bed or a sliding bed, depending on the particle diameter and the pipe diameter and of course the liquid properties.

The DHLLDV framework divides particles in 5 regions and a lower limit. These regions are for sand and gravel; very small particles up to about $\delta_{v}$, small particles up to $0.2 \mathrm{~mm}$, a transition region from $0.2 \mathrm{~mm}$ to $2 \mathrm{~mm}$, particles larger than $2 \mathrm{~mm}$ and particles larger than 0.015 times the pipe diameter. The lower limit is the transition between a sliding bed and heterogeneous transport.
Each region is modelled separately, resulting in dynamic transition particle diameters, depending on the parameters involved. The modelling is independent of head loss models. The modelling is calibrated mainly with sand and gravel experimental data, although limited data is used for other relative submerged densities.

The equations found give an upper limit to the LDV and are thus conservative, but safe.

Although the model seems completed, there are still a number of issues that required further investigation. For region 1, very fine particles, the liquid viscosity and density may need adjustment, however it is the question whether this is necessary in the viscous sub-layer. For region 5, very large particles, it is the question whether an LDV exists. At concentrations above a certain threshold (related to sheet flow) the bed concentration may decrease continuously. Still some characteristic LDV value is required to determine the slip velocity in the DHLLDV framework. For all regions the relative submerged density has 


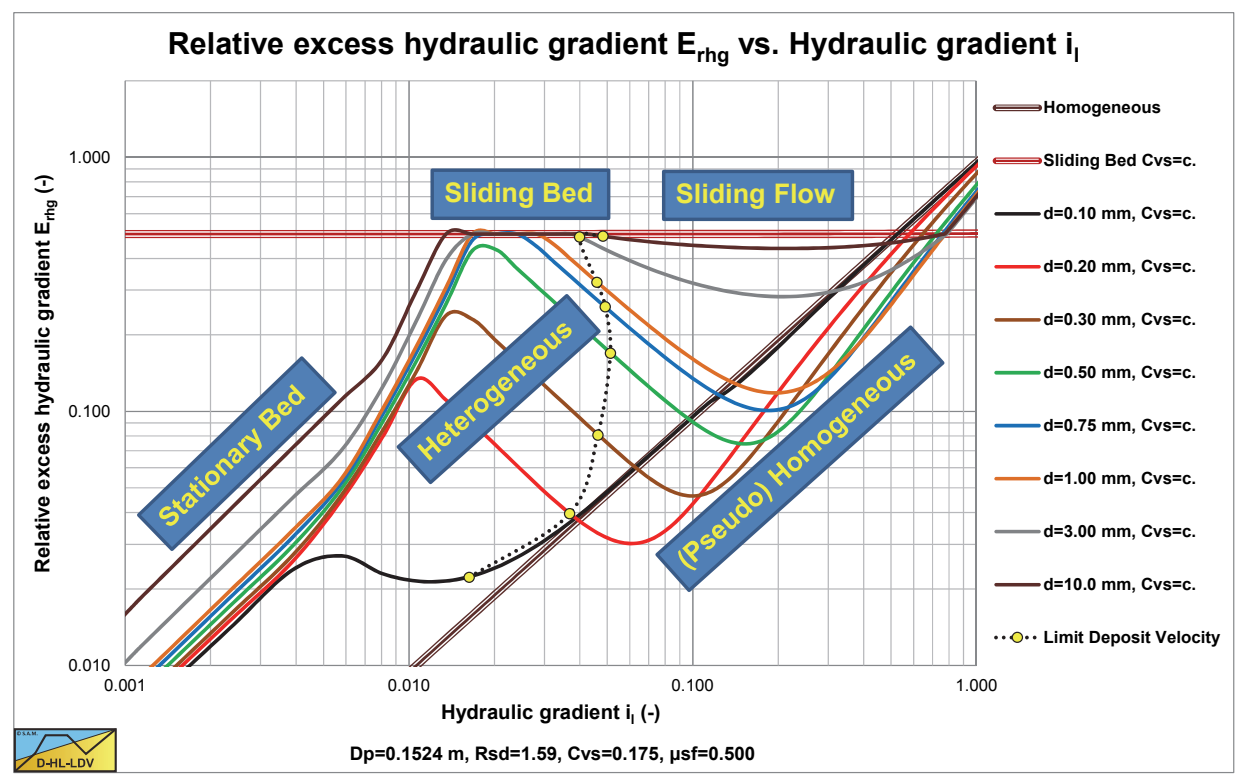

Fig. 8. The path of the LDV curve in a $E_{r h g}$ graph for a 6 inch $(0.1524 \mathrm{~m})$ pipe.

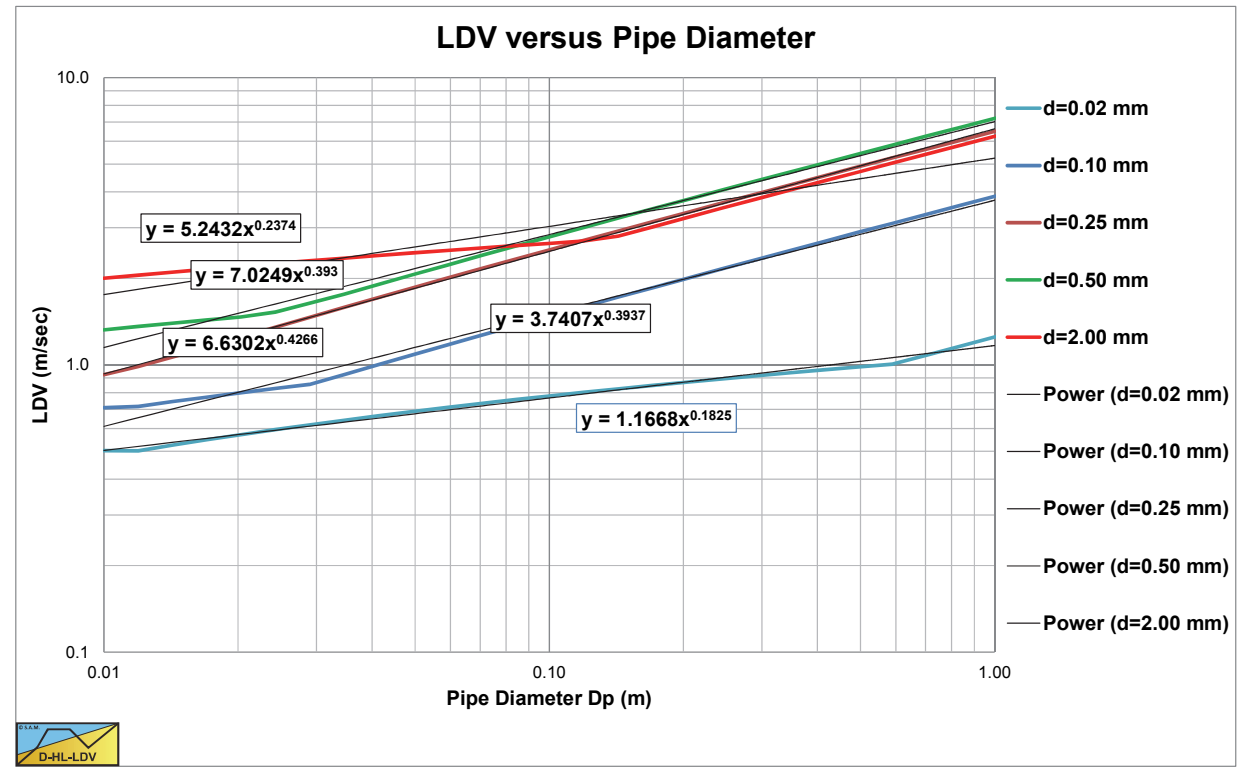

Fig. 9. The LDV as a function of the pipe diameter for 5 particle sizes.

not yet been investigated thoroughly, although it is part of the model. For the limited data investigated the model gives good results, but more experimental data could adjust the implementation of the relative submerged density. For dredging purposes however the model is suitable.

More graphs, spreadsheets and programs can be found on the website of The Delft Head Loss \& Limit Deposit Velocity Framework: $\underline{w w w . d h l l d v . c o m}$.

\section{REFERENCES}

Azamathulla, H.M., Ahmad, Z., 2013. Estimation of critical velocity for slurry transport through pipeline using adaptive neuro-fuzzy interference system and gene-expression programming. Journal of Pipeline Systems Engineering and Practice, 131-137.
Berg, C.H., 1998. Pipelines as transportation systems. In: Proceedings of the European Mining Course. IHC-MTI, Kinderdijk, the Netherlands.

Charles, M.E., 1970. Transport of solids by pipeline. Hydrotransport 1. BHRA, Cranfield.

Davies, J.T., 1987. Calculation of critical velocities to maintain solids in suspension in horizontal pipes. Chemical Engineering Science, 42, 7, 1667-1670.

Durand, R., 1953. Basic Relationships of the Transportation of Solids in Pipes - Experimental Research. In: Proceedings of the International Association of Hydraulic Research. Minneapolis.

Durand, R., Condolios, E., 1952. Etude experimentale du refoulement des materieaux en conduites en particulier des produits de dragage et des schlamms. [Experimental study of the discharge pipes materieaux especially products of 
dredging and slurries]. Deuxiemes Journees de l'Hydraulique, 27-55. (In French.)

Fuhrboter, A., 1961. Über die Förderung von Sand-WasserGemischen in Rohrleitungen. [On the advances of sand water mixtures in pipelines]. Mitteilungen des FranziusInstituts, H. 19.(In German.)

Garcia, M.H., 2008. Sedimentation Engineering (Vol. 110). ASCE Manuals \& Reports on Engineering Practise No. 110.

Gibert, R., 1960. Transport hydraulique et refoulement des mixtures en conduites. [Hydraulic transport and discharge pipes of mixtures]. Annales des Ponts et Chausees, 130, 3, 307-374, 130, 4, 437-494. (In French.)

Gillies, R.G., 1993. Pipeline flow of coarse particles, $\mathrm{PhD}$ Thesis. University of Saskatchewan, Saskatoon.

Gogus, M., Kokpinar, M.A., 1993. Determination of critical flow velocity in slurry transporting pipeline systems. In: Proceeding of the 12th International Conference on Slurry Handling and Pipeline Transport. British Hydraulic Research Group, Bedfordshire, UK, pp. 743-757.

Graf, W.H., Robinson, M., Yucel, O., 1970. The critical deposit velocity for solid-liquid mixtures. Hydrotransport 1. BHRA, Cranfield, UK, pp. H5-77-H5-88.

Hepy, F.M., Ahmad, Z., Kansal, M.L., 2008. Critical velocity for slurry transport through pipeline. Dam Engineering, 19, $3,169-184$.

Jufin, A.P., Lopatin, N.A., 1966. O projekte TUiN na gidrotransport zernistych materialov po stalnym truboprovodam. [TUiN project on hydrotransport of grain materials in steel tubes]. Gidrotechniceskoe Strojitelstvo, 9, 49-52. (In Russian.)

Kokpinar, M.A., Gogus, M., 2001. Critical velocity in slurry transport in horizontal pipelines. Journal of Hydraulic Engineering, 127, 9, 763-771.

Lahiri, S.K., 2009. Study on slurry flow modelling in pipeline. National Institute of Technology, Durgapur, India.

Miedema, S.A., 2012a. Constructing the Shields Curve: Part A Fundamentals of the Sliding, Rolling and Lifting Mechanisms for the Entrainment of Particles. Journal of Dredging Engineering, 12., 1-49.

Miedema, S.A., 2012b. Constructing the Shields Curve: Part B Sensitivity Analysis, Exposure \& Protrusion Levels, Settling Velocity, Shear Stress \& Friction Miedema, S.A., 2014 Velocity, Erosion Flux and Laminar Main Flow. Journal of Dredging Engineering, 12, 50-92.

Miedema, S.A., 2014. An analytical approach to explain the Fuhrboter equation. Maritime Engineering, 167, 2, 1-14.

Miedema, S.A., 2015a. A head loss model for homogeneous slurry transport. Journal of Hydrology and Hydromechanics, $1,1-12$.

Miedema, S.A., 2015b. Head loss model for slurry transport in the heterogeneous regime. Journal of Ocean Engineering, 12., 50-92.

Miedema, S.A., 2015c. The Slip Ratio or Holdup Function in Slurry Transport. Dredging Summit and Expo 2015. WEDA, Houston, Texas, USA, p. 12.

Miedema, S.A., Matousek, V., 2014. An explicit formulation of bed friction factor for sheet flow. In: Proc. $15^{\text {th }}$ International Freight Pipeline Society Symposium, IFPS, Prague, Czech Republic, p. 17.

Miedema, S.A., Ramsdell, R.C., 2013. A head loss model for slurry transport based on energy considerations. In: Proc. XX World Dredging Conference, WODA, Brussels, Belgium, p. 14.
Miedema, S.A., Ramsdell, R.C., 2014a. An analysis of the hydrostatic approach of wilson for the friction of a sliding bed. WEDA/TAMU. WEDA, Toronto, Canada, p. 21.

Miedema, S.A., Ramsdell, R.C., 2014b. The Delft Head Loss \& Limit Deposit Velocity Model. In: Hydrotransport, BHR Group, Denver, USA, p. 15.

Newitt, D.M., Richardson, M.C., Abbott, M., Turtle, R.B., 1955. Hydraulic conveying of solids in horizontal pipes. Transactions of the Institution of Chemical Engineers, 33, 93-110.

Oroskar, A.R., Turian, R.M., 1980. The hold up in pipeline flow of slurries. AIChE, 26, 550-558.

Parzonka, W., Kenchington, J.M., Charles, M.E., 1981. Hydrotransport of solids in horizontal pipes: Effects of solids concentration and particle size on the deposit velocity. Canadian Journal of Chemical Engineering, 59, 291-296.

Poloski, A.P., Etchells, A.W., Chun, J., Adkins, H.E., Casella, A.M., Minette, M.J., Yokuda, S., 2010. A pipeline transport correlation for slurries with small but dense particles. Canadian Journal of Chemical Engineering, 88, 182-189.

Ramsdell, R.C., Miedema, S.A., 2013. An overview of flow regimes describing slurry transport. In: WODCON XX, WODA, Brussels, Belgium, p. 15.

Sanders, R.S., Sun, R., Gillies, R.G., McKibben, M., Litzenberger, C., Shook, C.A., 2004. Deposition velocities for particles of intermediate size in turbulent flows. In: Hydrotransport 16, BHR Group, Santiago, Chile, pp. 429442.

Schiller, R.E., Herbich, J.B., 1991. Sediment Transport in Pipes. Handbook of Dredging. McGraw-Hill, New York.

Shook, C.A., Gillies, R.G., Sanders, R.S., 2002. Pipeline Hydrotransport with Application in the Oil Sand Industry. SRC Publication 11508-1E02, Saskatchewan Research Council, Saskatoon, Canada.

Souza Pinto, T.C., Moraes Junior, D., Slatter, P.T., Leal Filho, L.S., 2014. Modelling the critical velocity for heterogeneous flow of mineral slurries. International Journal of Multiphase Flow, 65, 31-37.

Thomas, A.D., 1979. Predicting the deposit velocity for horizontal turbulent pipe flow of slurries. International Journal of Multiphase Flow, 5, 113-129.

Thomas, A.D., 2014. Slurries of most interest to the mining industry flow homogeneously and the deposit velocity is the key parameter. In: HydroTransport 19, BHR Group, Denver, Colorado, USA, pp. 239-252.

Thomas, D.G., 1962. Transport characteristics of suspensions: Part VI. Minimum velocity for large particle size suspensions in round horizontal pipes. A.I.Ch.E. Journal, 8, 3, 373-378.

Thomas, D.G., 1965. Transport characteristics of suspensions: VIII. A note on the viscosity of Newtonian suspensions of uniform spherical particles. Journal of Colloidal Sciences, 20, 267-277.

Turian, R.M., Hsu, F. L., Ma, T.W., 1987. Estimation of the critical velocity in pipeline flow of slurries. Powder Technology, 51, 35-47.

Wasp, E.J., Slatter, P.T., 2004. Deposition velocities for small particles in large pipes. In: Proc. 12th International Conference on Transport \& Sedimentation of Solid Particles, Prague, Czech Republic, pp. 20-24.

Wasp, E.J., Kenny, J.P., Aude, T.C., Seiter, R.H., Jacques, R.B., 1970. Deposition velocities transition velocities and spatial distribution of solids in slurry pipelines. In: Hydro Transport 1, paper H42, BHRA Fluid Engineering, Coventry, pp. 53-76. 
Wasp, E.J., Kenny, J.P., Gandhi, R.L., 1977. Solid liquid flow slurry pipeline transportation. Transactions Technical Publications.

Wilson, K.C., 1979. Deposition limit nomograms for particles of various densities in pipeline flow. In: Hydrotransport 6, BHRA, Canterbury, UK, p. 12.

Wilson, K.C., Judge, D.G., 1976. New techniques for the scaleup of pilot plant results to coal slurry pipelines. In: Proceedings International Symposium on Freight Pipelines, University of Pensylvania, Washington DC, USA, pp. 1-29.

Wilson, K.C., Judge, D.G., 1977. Application of analytical model to stationary deposit limit in sand water slurries. In: Dredging Technology, BHRA Fluid Engineering, College Station, Texas, USA, pp. J1 1-12.

Wilson, K.C., Addie, G.R., Clift, R., 1992. Slurry Transport using Centrifugal Pumps. Elsevier Applied Sciences, New York.

Wilson, W.E., 1942. Mechanics of flow with non colloidal inert solids. Transactions ASCE, 107, 1576-1594.

Yagi, T., Okude, T., Miyazaki, S., Koreishi, A., 1972. An Analysis of the Hydraulic Transport of Solids in Horizontal Pipes. Nagase, Yokosuka, Japan. Report of the Port \& Harbour Research Institute, Vol. 11, No. 3.

Zandi, I., Govatos, G., 1967. Heterogeneous flow of solids in pipelines. Proc. ACSE, J. Hydraul. Div., 93(HY3), 145-159.

\section{NOMENCLATURE}

$C_{v b} \quad$ Bed volumetric concentration

$C_{v s} \quad$ Spatial volumetric concentration

$C_{v s, l d v} \quad$ Concentration of bed at LDV

$C_{v r, l d v} \quad$ Bed fraction at LDV

$C_{v t} \quad$ Transport or delivered volumetric concentration
$C_{x}$

$d$

$d_{0}$

$D_{p}$

$E_{r h g}$

$F_{L}$

$F_{s f}$

$F_{W}$

$g$

$h$

$i_{l}$

$i_{m}$

$\Delta L$

$n$

$R_{s d}$

$u_{*}$

$v_{l s}$

$v_{l s, l d v}$

$v_{t}$

$\alpha_{p}$

$\delta_{v}$

$\lambda_{l}$

$\kappa_{C}$

$\rho_{l}$

$v_{l}$

$\mu_{s f}$

$\tau_{12}$
Durand \& Condolios coefficient

Particle diameter

Transition particle diameter

Pipe diameter

Relative excess hydraulic gradient

Durand Limit Deposit Velocity Froude number

Sliding friction force

Weight of bed at LDV

Gravitational constant (9.81)

Thickness of bed layer at LDV

Hydraulic gradient of liquid

Hydraulic gradient of mixture

Length of pipeline

Porosity of bed

Relative submerged density

Friction velocity

Cross-section averaged line speed

Line speed at Limit Deposit Velocity

Particle terminal settling velocity

Energy fraction coefficient

Thickness of viscous sub layer

Darcy-Weisbach friction factor between

liquid and pipe wall

Concentration eccentricity constant

Density of liquid

Kinematic viscosity liquid

Sliding friction coefficient

Bed shear stress $\mathrm{m} / \mathrm{s}^{2}$

$\mathrm{m}$

Received 11 November 2014 Accepted 15 June 2015 